Relations industrielles

Industrial Relations

\title{
Action politique et syndicats
}

\section{Gérard Filion}

Volume 15, numéro 4, octobre 1960

URI : https://id.erudit.org/iderudit/1021946ar

DOI : https://doi.org/10.7202/1021946ar

Aller au sommaire du numéro

\section{Éditeur(s)}

Département des relations industrielles de l’Université Laval

\section{ISSN}

0034-379X (imprimé)

1703-8138 (numérique)

Découvrir la revue

Citer ce document

Filion, G. (1960). Action politique et syndicats. Relations industrielles / Industrial Relations, 15(4), 496-499. https://doi.org/10.7202/1021946ar

Tous droits réservés @ Département des relations industrielles de l’Université Laval, 1960
Ce document est protégé par la loi sur le droit d'auteur. L’utilisation des services d'Érudit (y compris la reproduction) est assujettie à sa politique d'utilisation que vous pouvez consulter en ligne.

https://apropos.erudit.org/fr/usagers/politique-dutilisation/ 


\section{ACTION POLITIQUE ET SYNDICATS}

\section{GÉrard FiLIon}

Les syndicats ont-ils le droit de chercher à influencer la politique? Il reste peu de gens pour le contester. Ils ne font en cela qu'imiter les autres groupements professionnels. Les chambres de commerce, les associations de patrons, les groupements agricoles, les associations d'hommes de profession, les fédérations de coopératives, s'emploient à convaincre l'autorité publique de faire ou de ne pas faire certaines choses. Les moyens qu'ils emploient sont variés: mémoires, entrevues, lettres et télégrammes, dénonciations, menaces. Que certaines interventions soient inopportunes ou démagogiques, personne ne le conteste; chaque groupement est enclin à confondre son intérêt particulier avec le bien commun. Mais il semble qu'à défaut de mieux, c'est encore de cette façon que le régime démocratique conserve un minimum de vigilance. Donc l'attention politique indirecte, qui cherche à faire pression sur les autorités ou à convaincre l'opinion publique, est légitime pour les syndicats de travailleurs comme pour les autres groupements professionnels.

Est-il légitime d'appuyer un parti à l'exclusion des autres, de s'y affilier, de le financer, d'inciter ses membres à lui accorder leurs suffrages?

Pour répondre à cette question, il faut faire un tout petit peu de philosophie.

Qu'est-ce qu'un parti et qu'est-ce qu'un syndicat?

Un parti est un groupement d'hommes qui se proposent de servir le bien commun en prenant la direction des affaires publiques dans une cité, une province ou un pays. Le parti est d'ordre général, il s'adresse au public.

Le syndicat se réfère à une catégorie professionnelle; il s'adresse à un groupe plus ou moins nombreux d'individus exerçant un métier commun; son objet est économico-social et sa fin, l'intérêt professionnel de ses membres.

Du fait que le parti et le syndicat sont deux entités distinctes, que leur objet est différent et qu'ils poursuivent chacun une fin propre, s'ensuit-il que toute collaboration organique entre les deux soit illégitime? Il faudrait peut-être distinguer.

Si le syndicat prétend substituer l'intérêt particulier qu'il représente au bien commun de toute la société, je serais porté à émettre des doutes sur sa légitimité. Un parti de classes, qui prétend accéder à la direction des affaires publiques pour imposer à la vie politique des moyens et des objectifs de classe, ne représente vraiment pas le bien commun.

Mais il n'existe pas d'incompatibilité de nature entre la fin d'un syndicat et la fin d'un parti politique. Mgr Pietro Pavan, vice-président des Semaines Sociales d'Italie, s'exprime d'une façon très claire sur le sujet: «Peuple, parti politique, bien commun; catégorie professionnelle, syndicat, intérêts professionnels. On peut dire en un certain sens, que le parti est au peuple, ce que le syndicat est à la catégorie professionnelle. Et on peut affirmer qu'entre le parti et le bien commun, il y a un rapport analogue tout comme entre le syndicat et les intérêts profession- 
nels. La catégorie professionnelle se distingue du peuple au moins comme la partie du tout. D'autre part, si le peuple est un organisme vivant, la catégorie professionnelle est un tissu vital; de façon analogue, les intérêts professionnels, en quantité et en qualité, se distinguent du bien commun, bien qu'en fait, ils sont et ils doivent être considérés comme des éléments essentiels de ce même bien commun $»$.

Je mettrai cependant certaines conditions à l'action légitime des syndicats en faveur d'un parti politique:

1) Il faut que cette action ne nuise pas à la poursuite du bien particulier du syndicat. Si l'adhésion officielle à un parti devait faire naître des luttes intestines, des divisions et même une certaine scission, cette action politique deviendrait illégitime parce qu'elle mettrait en danger la fin immédiate du syndicat.

2) Il ne faut pas que le syndicat se transforme petit à petit en parti politique, ou qu'il soit lié de si près au parti qu'il soit incapable de s'en dégager. Le danger se fera sentir quand, d'aventure, le parti sera porté au pouvoir. Le syndicat sera alors exposé à devenir l'instrument du parti, puis l'organe de l'Etat. Or le syndicat est une institution d'ordre privé qui poursuit un bien particulier; quand il se fait absorber par les structures de l'Etat, il perd sa liberté et en même temps sa raison d'être.

A ces conditions, je ne vois rien d'illégitime à ce qu'un syndicat appuie officiellement de ses votes et de ses deniers un parti politique qui lui paraît capable d'assurer le bien commun de la société civile.

Dans ce contexte économico-social où nous vivons, le geste est-il souhaitable? Plusieurs soutiendront la négative. A l'appui de leur thèse, ils feront la longue énumération de mesures sociales que des partis soi-disant peu démocratiques dans leurs structures et capitalistes dans leur inspiration ont consenties durant les dernières décennies: protection de la femme au travail et interdiction du travail des enfants, limitation des heures de travail et mesures d'hygiène dans les usines, protection contre les maladies professionnelles, les accidents, le chômage, assistance en cas d'infortune et de vieillesse, primes aux mariages et aux naissances, et le reste, et le reste. L'énumération est impressionnante.

Comment faire le partage des responsabilités actives et passives de ce qui s'est fait depuis le début du siècle? Les syndiqués affirmeront qu'ils ont arraché par bribes les mesures de législation sociale dont nous jouissons, et ce n'est pas complètement faux. Les partis sociaux se vanteront d'avoir imposé par leur présence dans les parlements le souci d'une meilleure protection des travailleurs, et ils n'ont pas complètement tort. Les défenseurs des vieux partis rétorqueront que chaque pièce de législation est venue en son temps, quand la productivité a permis aux entreprises d'assumer une charge supplémentaire et que de toute façon, avec ou sans syndicats, avec ou sans partis sociaux, ça serait venu quand même; prenons l'affirmation avec un grain de sel, mais reconnaissons qu'elle contient une partie de vrai.

Mais enfin la situation étant ce qu'elle est, un parti démocratique d'inspiration populaire a-t-il encore un objet dans le milieu où nous vivons? 
La société est formée d'hommes de tempéraments divers, d'opinions variées. Les uns favorisent le mouvement. Ils regardent en avant, ils cherchent de nouvelles formes d'activités, des modes nouveaux de penser et de vivre. D'autres se barricadent dans la stabilité, se reposent sur l'acquis, se méfient des innovations, ce qui a fait ses preuves n'a pas de raison de changer! Les deux attitudes sent saines, elles se complètent. Il est bon qu'elles s'expriment dans la vie politique, comme en littérature et en art. Quand les forces conservatrices sont trop puissantes, les éléments jeunes et dynamiques s'irritent, disent et font des bêtises. Quand les forces progressistes sont lâchées, si on me permet cette image baroque, la bride sur le cou, c'est l'anarchie intellectuelle et morale, et c'est la révolution politique. Une société sainement équilibrée donne une chance égale aux deux tendances.

C'est pourquoi, même si un parti populaire ne poursuit plus les mêmes objectifs impérieux qu'il aurait ambitionné il y a une génération, sa présence est un élément d'équilibre et une garantie de saine démocratie. Au moins les éléments jeunes, les esprits inquiets, les tempéraments dynamiques y trouveront à s'employer dans l'ordre.

Et puis, un parti populaire n'aurait d'autre objet que d'assurer une démocratisation plus prononcée de la politique qu'il aurait déjà sa raison d'être dans le milieu canadien.

\section{Est-ce réalisable?}

Sur une modeste échelle, évidemment oui. Mais un parti ambitionne plus que de siéger indéfiniment dans l'opposition. C'est le pouvoir qui est l'objet de ses ambitions. Gardons-nous de jouer au diseur de bonne aventure. Constatons simplement que le PSD, pour ne pas le nommer, a connu une fortune variable, mais qu'il n'a pas encore, après vingt-sept ans, atteint son objectif, sauf en Saskatchewan. L'appui des syndicats et des syndiqués lui donnera-t-il le stimulant dont il a besoin pour s'engager dans le deuxième tour de piste?

Je constate simplement que les syndiqués ne pensent pas toujours et ne votent pas toujours comme leurs chefs. La conscience de classe ne dépasse guère le champ limité de la discussion et de la signature d'un contrat de travail. Au-delà de cet objectif important mais limité, les spéculations sur la conception que l'on doit avoir de l'Etat et de son gouvernement ne sont pas des sujets de conversation courante.

Je constate en deuxième lieu que le nouveau parti, ne voulant pas être un parti de classe, ce dont il faut le féliciter, s'ouvre largement à tous les esprits libéraux, ce qui veut dire passablement de gens qui ne sont pas tous des travailleurs d'usine ni des agriculteurs. Cette invasion de collets-blancs, d'esprits plus bourgeois qu'ils ne veulent le laisser voir, fera-t-elle dériver vers la droite le parti à fonder? Ce n'est pas impossible. Aussi peut-on prévoir d'avance qu'il y aura dans le nouveau parti une gauche, un centre et une droite, la gauche étant représentée par la vieille garde CCF encore fidèle au programme de Régina de 1933, et la droite par les éléments petit-bourgeois et les syndicats d'ouvriers spécialisés qui groupent parfois des gens aussi conservateurs que leurs patrons. 
L'action politique des syndicats est légitime à certaines conditions, même leur adhésion ouverte à un parti n'est pas plus blâmable que l'adhésion clandestine d'autres groupements aux vieux partis traditionnels. Est-elle souhaitable? Oni, mais pas avec la même urgence qu'il y a une ou deux générations. Est-elle réalisable? Le succès complet, c'est-à-dire la prise du pouvoir, n'est pas pour demain ni après-demain; plus tard on verra.

\section{L'EGLISE ET L'ORGANISATION PROFESSIONNELLF.}

Jean-Guy Hamelin, ptre

Le theme de l'Organisation professionnelle tient une place de premier plan dans l'Enseignement social de l'Eglise. Les divers commentateurs des documents pontificaux ont vu dans l'Organisation professionnelle «l'idée maîtresse de la doctrine sociale catholique sur la restauration de l'ordre économique $\diamond$. * Nous ne nous lassons pas, écrivait Pie XII aux délégués du Mouvement ouvrier chrétien belge, le 11 septembre 1949, de recommander instamment l'élaboration d'un statut de droit public de la vie économique, de toute la vie sociale en général, selon l'Organisation professionnelle ». 1

Evidemment, lorsque nous parlons ici du mot \&profession》, il ne faut pas l'entendre au sens étroit de «métier». Il faut plutôt dire qu'il s'agit de branches de l'activité économique, de secteurs industriels. ${ }^{2}$ Le fait pour un groupe d'hommes de s'appliquer au même genre d'opérations ou de participer par des métiers différents à la même branche de production fait d'eux comme un corps social naturel qui a ses intérêts communs, établit entre eux un lien naturel, un centre de relations. La détermination de l'unité à organiser professionnellement devrait donc admettre certaines considérations sociologiques qui peuvent en élargir plus on moins les cadres: on comprend pourquoi les Souverains Pontifes n'ont jamais fixé les limites de ce qu'ils entendaient par «profession».

Les grands papes de la question sociale ont mis en évidence les principes qui devraient guider les hommes dans leur travail pour l'édification d'une société économique juste et ordonnée.

Léon XIII d'abord met fortement en lumière le droit et le devoir pour lesouvriers de s'associer. Cependant, il ne parle pas expressément de l'Organisation. professionnelle: il a surtout en vue d'encourager des sociétés composées soit des seuls ouvriers, soit des sociétés mixtes comprenant patrons et ouvriers (syndicats mixtes). Ce qui domine chez lui, c'est l'importance de l'association.

Avec Pie XI, la doctrine sociale de l'Eglise fait un pas considérable, nous dirions historique: le pape demande que la profession soit considérée comme un corps organisé dans l'Etat selon la fonction supplétive des collectivités. Il établit

(1) Relations humaines et société contemporaine, Utz. no 3264.

(2) Voilà pourquoi une autre terminologie serait peut-être plus adéquate pour désigner cette structure de la vie économique qu'est l'Organisation professionnelle. 